$\underset{1}{9}$ DIAGNOStIC PATHOLOGY

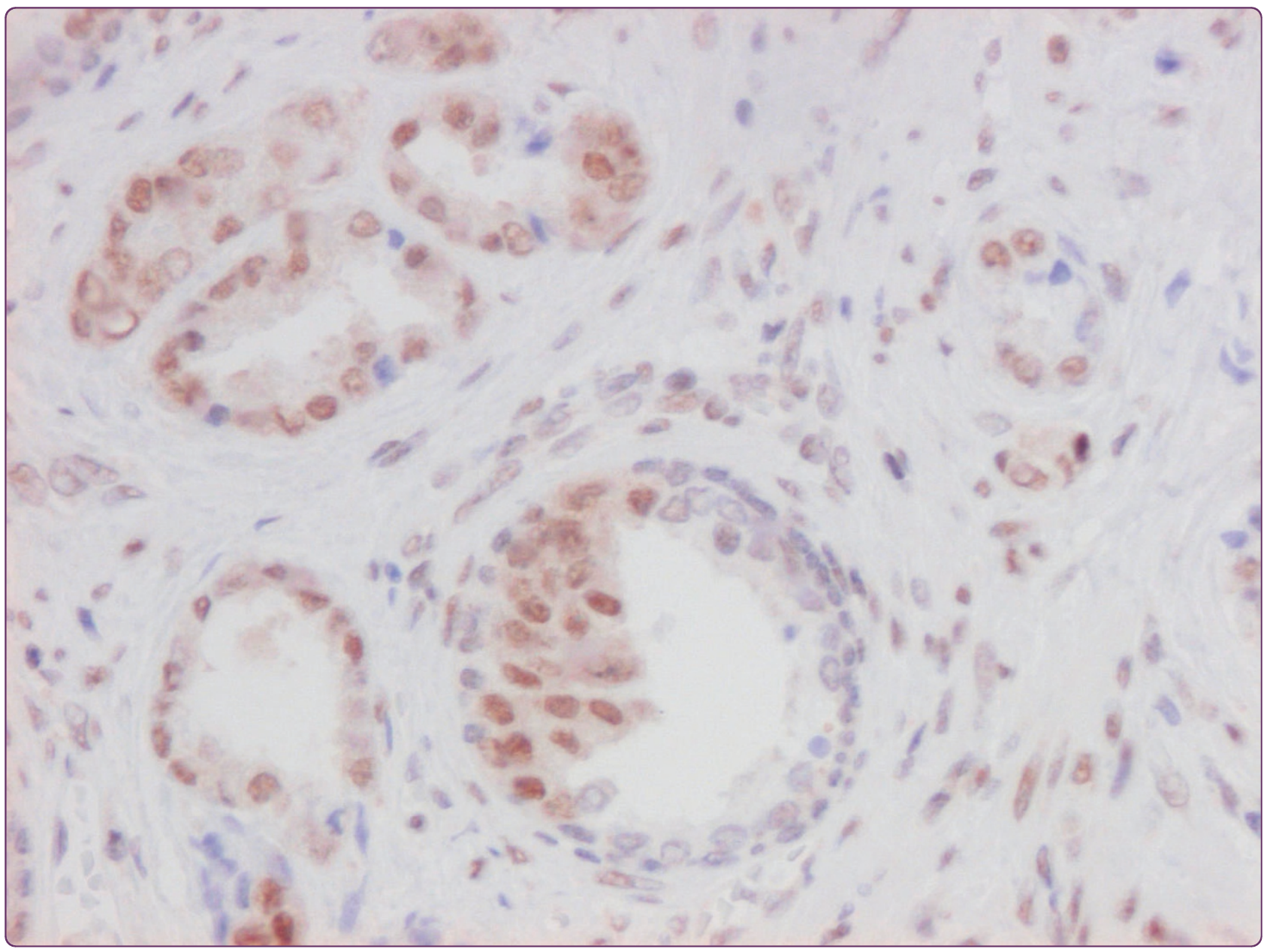

High RBM3 expression in prostate cancer independently predicts a reduced risk of biochemical recurrence and disease progression

Jonsson et al. 


\title{
High RBM3 expression in prostate cancer independently predicts a reduced risk of biochemical recurrence and disease progression
}

\author{
Liv Jonsson ${ }^{1 *}$, Alexander Gaber ${ }^{1}$, David Ulmert ${ }^{2}$, Mathias Uhlén ${ }^{3,4}$, Anders Bjartell ${ }^{2}$ and Karin Jirström
}

\begin{abstract}
Background: High expression of the RNA-binding protein RBM3 has previously been found to be associated with good prognosis in breast cancer, ovarian cancer, malignant melanoma and colorectal cancer. The aim of this study was to examine the prognostic impact of immunohistochemical RBM3 expression in prostate cancer.

Findings: Immunohistochemical RBM3 expression was examined in a tissue microarray with malignant and benign prostatic specimens from 88 patients treated with radical prostatectomy for localized disease. While rarely expressed in benign prostate gland epithelium, RBM3 was found to be up-regulated in prostate intraepithelial neoplasia and present in various fractions and intensities in invasive prostate cancer. High nuclear RBM3 expression was significantly associated with a prolonged time to biochemical recurrence (BCR) (HR 0.56, 95\% Cl: 0.34-0.93, $p=$ 0.024 ) and clinical progression (HR 0.09, 95\% Cl: 0.01-0.71, $p=0.021$ ). These associations remained significant in multivariate analysis, adjusted for preoperative PSA level in blood, pathological Gleason score and presence or absence of extracapsular extension, seminal vesicle invasion and positive surgical margin (HR 0.41, 95\% Cl: 0.19$0.89, p=0.024$ for BCR and HR 0.06, 95\% Cl: 0.01-0.50, $p=0.009$ for clinical progression).

Conclusion: Our results demonstrate that high nuclear expression of RBM3 in prostate cancer is associated with a prolonged time to disease progression and, thus, a potential biomarker of favourable prognosis. The value of RBM3 for prognostication, treatment stratification and follow-up of prostate cancer patients should be further validated in larger studies.
\end{abstract}

Keywords: RBM3, immunohistochemistry, prognosis, prostate cancer

\section{Findings}

Background and hypothesis

Prostate cancer is the leading cause of cancer death in men in economically developed countries [1] and there was an estimated 328000 cases in Europe in 2008 which makes it the most common form of cancer in men [2]. For localized cancer, radical prostatectomy is the most common treatment and it has shown a benefit in cancer specific survival in comparison to watchful waiting [3]. Nevertheless, in some cases the cancer will recur with detectable prostate specific antigen (PSA) concentrations in blood, known as biochemical recurrence (BCR) [4].

\footnotetext{
* Correspondence: Liv.Jonsson@med.lu.se

'Department of Clinical Sciences, Pathology, Lund University, Skåne

University Hospital, 22185 Lund, Sweden Full list of author information is available at the end of the article
}

Apart from PSA, no diagnostic or prognostic biomarkers have yet been incorporated into clinical protocols for management and risk stratification of prostate cancer patients despite extensive research efforts $[5,6]$. Thus, there is still a great need for new biomarkers to predict the course of the disease and differentiate indolent from life threatening cancer.

The RNA-binding motif protein 3, RBM3, is a glycine rich protein with an RNA recognition motif (RRM) capable of binding to both RNA and DNA and it is one of the earliest proteins synthesized in response to cold shock $[7,8]$. RBM3 is up-regulated in various types of human malignancies [9-11] and the role of RBM3 as a putative cancer biomarker was originally unravelled using an antibody-based discovery approach http://www.proteinatlas. org[12-14]. Since then, RBM3 protein expression, in
Ciomed Central 
particular its nuclear localization, has been demonstrated to be associated with a significantly improved survival in several cancer forms, e.g. breast cancer [11], ovarian cancer [15], malignant melanoma [16] and colorectal cancer (Hjelm et al, Proteomics Clinical Applications, in press). In ovarian cancer, RBM3 has also been demonstrated to be an independent factor of good prognosis at the gene expression level [15] and in vitro data point towards a link between RBM3 and cisplatin sensitivity in ovarian cancer cells [15], possibly by regulating several cellular processes involved in maintenance of DNA integrity [17].

In this study, we investigated the prognostic impact of RBM3 expression in prostate cancer by immunohistochemical (IHC) analysis of benign and malignant specimens from 88 patients treated with radical prostatectomy.

\section{Patients and methods}

The patient cohort consists of 88 patients, between 48-74 years, treated with radical prostatectomy for localized prostate cancer at Skåne University Hospital, Malmö, from 1998 - 2003. Patient and tumour characteristics are shown in Additional File 1. Written consent was obtained from the patients and the local ethics committee at Lund University approved the study. Histopathological, clinical and follow-up data were obtained from the clinical- and pathology records. Vital status and cause of death was obtained from the Swedish Cause of Death Registry up until December 2006. The mean follow up period was 58.5 months after which three patients had died and 85 patients were alive. BCR was defined as PSA $>0.2 \mathrm{ng} / \mathrm{ml}$ [18] with a confirmatory level and clinical progression included BCR, death from prostate cancer and adjuvant radiation therapy. During follow up, 14 (15.9\%) patients had BCR and $19(21.6 \%)$ patients had clinical progression. Five (5.7\%) patients received adjuvant radiotherapy and three (3.4\%) patients received adjuvant hormonal therapy.

For tissue microarray (TMA) construction, areas representing cancer and normal prostate tissue were marked on haematoxylin and eosin stained slides, whereby normal tissue was sampled in a noncancerous zone away from the tumour, in most cases the transition zone. Two $1.00 \mathrm{~mm}$ cores from areas with invasive cancer and one $1.00 \mathrm{~mm}$ core representing benign prostate tissue were sampled from each case and mounted in a new recipient block using a manual arraying device (MTA-1 Beecher Instruments Inc., Sun Prairie, WI, USA). For immunohistochemical analysis, $4 \mu \mathrm{m}$ TMA-sections were automatically pre-treated using the PT Link system and then stained in an Autostainer Plus (DAKO; Glostrup, Copenhagen, Denmark) with the mouse monoclonal anti-RBM3 antibody AAb030038; Atlas Antibodies AB, Stockholm, Sweden, diluted 1:5000. The specificity of the antibody has been validated previously [15]. RBM3 staining was evaluated by two independent observers ( $\mathrm{LJ}$ and $\mathrm{KJ}$ ) who were blinded to clinical and outcome data. The fraction of cells expressing RBM3 in the nucleus (NF) were denoted a score from 0 (0-1\%), 1 (2-25\%), 2 (26-50\%), 3 (51-75\%) and $4(>75 \%)$ and the intensity (NI) was scored as 0 (negative), 1 (weak), 2 (moderate) and 3 (strong). A combined nuclear score (NS) of NF $\times$ NI, was then constructed as previously described [15]. Cytoplasmic staining intensity was graded as 0 (no staining), 1 (weak staining) and 2 (strong staining).

\section{Statistical analysis}

Spearman's rho and Chi-square tests were used for analysis of the correlation between RBM3 expression and relevant clinicopathological characteristics. Kaplan-Meier analysis and log rank test were used to illustrate differences in BCR free time and progression free survival (PFS) according to RBM3 expression. For survival analyses, RBM3 expression (intensity $\times$ fraction) was dichotomized into weak vs strong using classification and regression tree analysis (CRT). Cox regression proportional hazards modelling were used to estimate the impact of RBM3 expression on BCR (with RBM3 as a continuous variable) and PFS (with RBM3 as a dichotomized variable) in both uniand multivariate analysis. All tests were two sided. A p-value of 0.05 was considered significant. All statistical analyses were performed using SPSS version 17 (SPSS Inc, Chicago, IL).

\section{Results}

While absent or weakly expressed in benign prostatic glands (Figure 1A), RBM3 was clearly up-regulated in prostatic intraepithelial neoplasia (PIN) (Figure 1B) and in invasive carcinoma RBM3 was expressed in various fractions and intensities (Figure 1C-E) with 33 (37.5\%) cases lacking RBM3 expression (Figure 2). A progression sequence with up-regulated RBM3 expression in PIN and retained high RBM3 expression in the invasive component is illustrated in Figure 3. There was no obvious heterogeneity in the staining pattern between duplicate tissue cores.

CRT analysis suggested an optimal cutoff point at NS > 2 to determine the impact of RBM3 expression on BCR free survival and PFS. While there was no association between RBM3 expression and conventional clinicopathological parameters (Additional File 2), Kaplan Meier analysis demonstrated that high expression of RBM3 was associated with a significantly prolonged time to BCR $(p=$ $0.004)$ and clinical progression $(p=0.004)$ (Figure 4$)$. These associations were confirmed in Cox univariate analysis (HR 0.56, 95\% CI: 0.34-0.93, $p=0.024$ for BCR and HR 0.09, 95\% CI: 0.01-0.71, $p=0.021$ for clinical progression) and remained significant in multivariate analysis, adjusted for preoperative PSA level, Gleason score and presence or absence of extracapsular extension, seminal vesicle invasion and positive surgical margins (HR 0.41, 

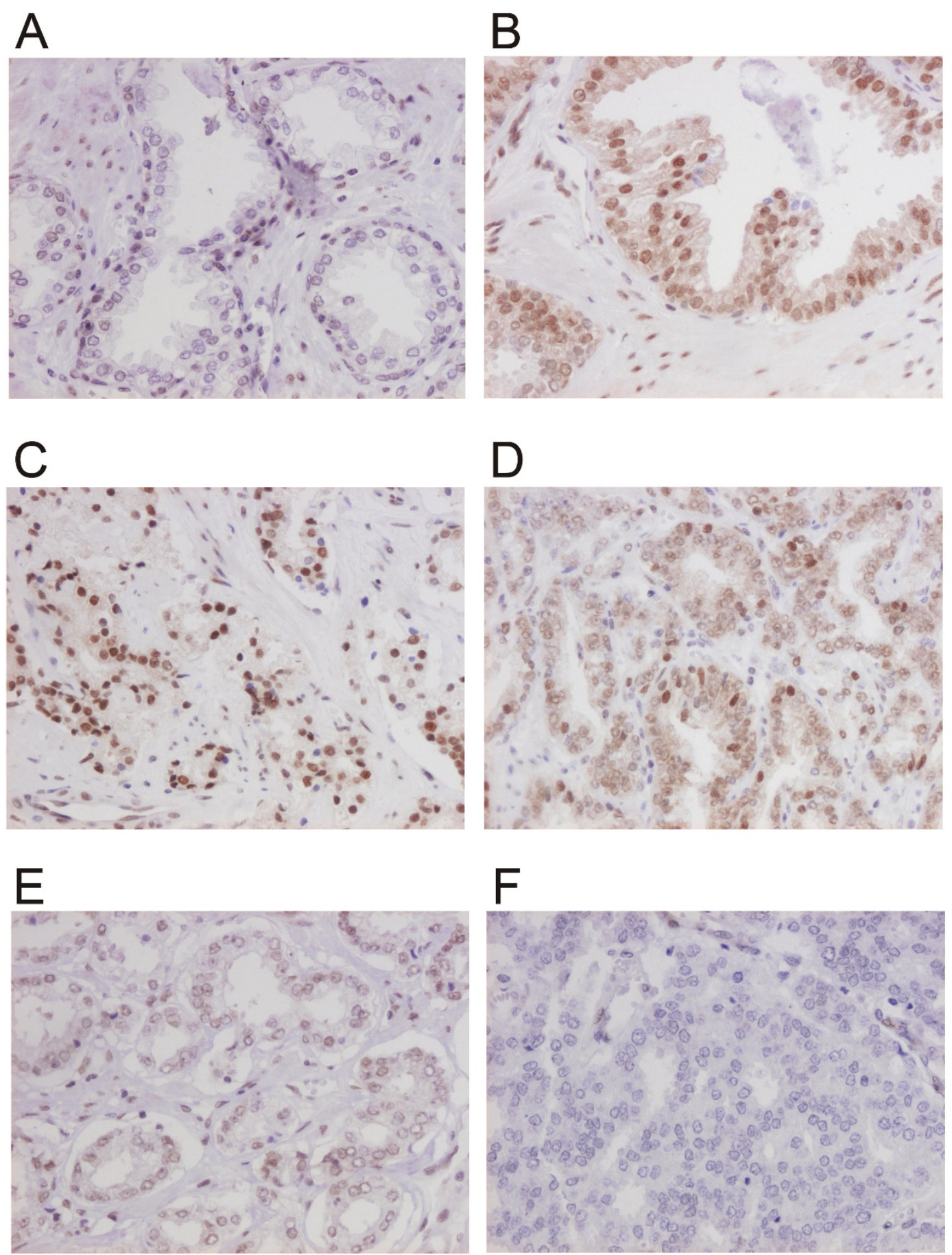

Figure 1 RBM3 expression in normal prostatic epithelium, prostatic intraepithelial neoplasia and in prostate cancer Immunohistochemical images of RBM3 expression in (A) normal prostatic epithelium, (B) prostatic intraepithelial neoplasia and invasive prostate cancer, ranging from (C) high, (D) moderate, (E) weak to (F) negative expression.

95\% CI: 0.19-0.89, $p=0.024$ for BCR and HR 0.06, 95\% CI: $0.01-0.50, p=0.009$ for clinical progression) (Table 1$)$. These significant associations were retained in both univariate and multivariate analysis also when the few patients who received adjuvant radiotherapy or hormonal therapy were excluded from the analysis (data not shown). Cytoplasmic RBM3 expression was not prognostic (data not shown). 


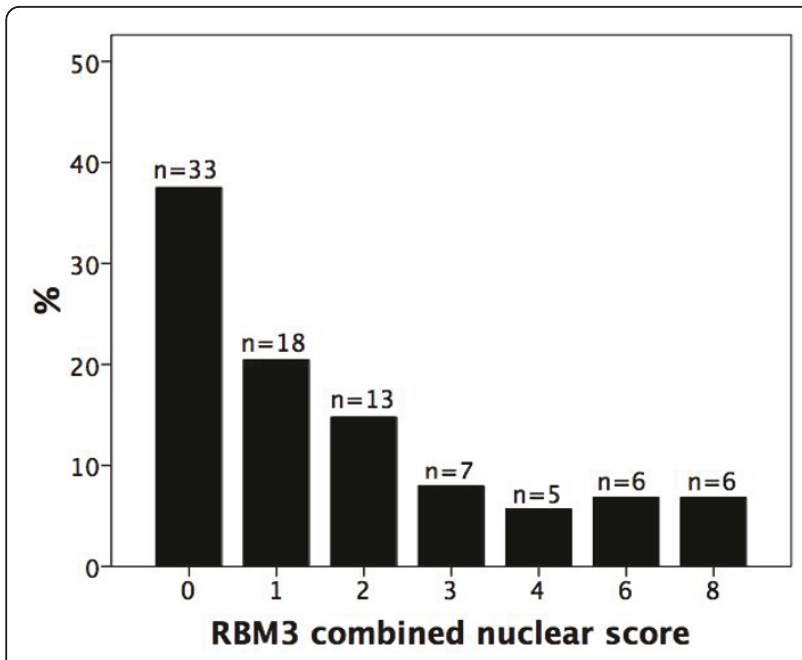

Figure 2 RBM3 staining distribution in prostate cancer. Distribution of nuclear RBM3 staining in invasive prostate cancer, denoted as nuclear score (fraction $x$ intensity).

\section{Interpretation and conclusions}

Our results demonstrate that RBM3, while rarely expressed in normal prostatic gland epithelium, is upregulated in PIN and invasive prostate cancer, and that patients with tumours expressing high nuclear levels of RMB3 have a significantly prolonged time to biochemical recurrence and clinical progression, also after adjustment for conventional prognostic factors. These findings are in line with previous findings in breast cancer [11], ovarian cancer [15], malignant melanoma [16] and colorectal cancer (Hjelm et al, Proteomics Clinical Applications, in press). Thus, there are increasing evidence for RBM3 being a biomarker of good prognosis in

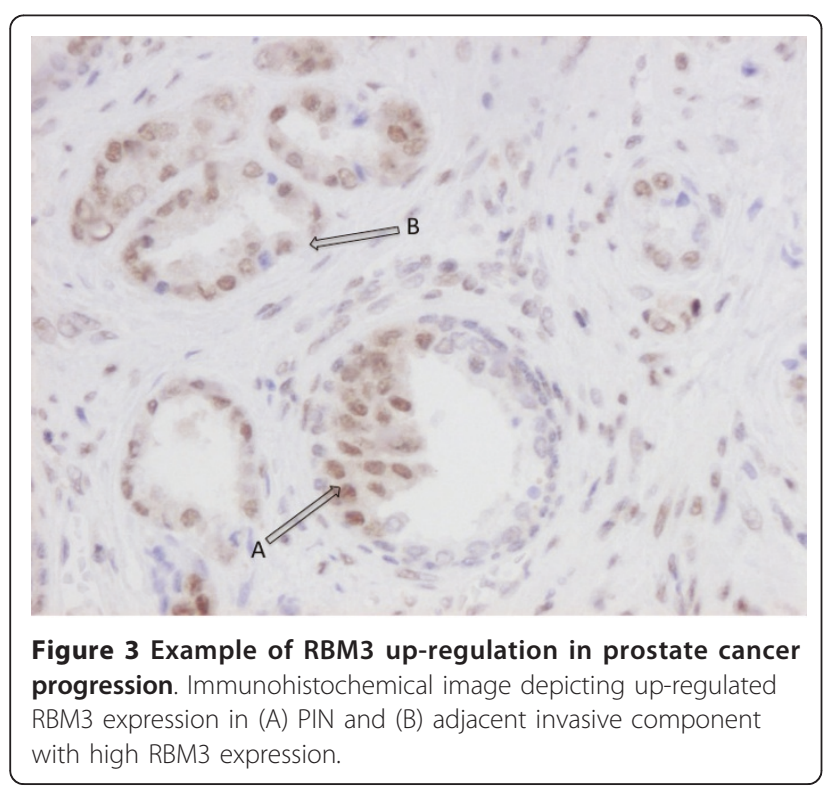

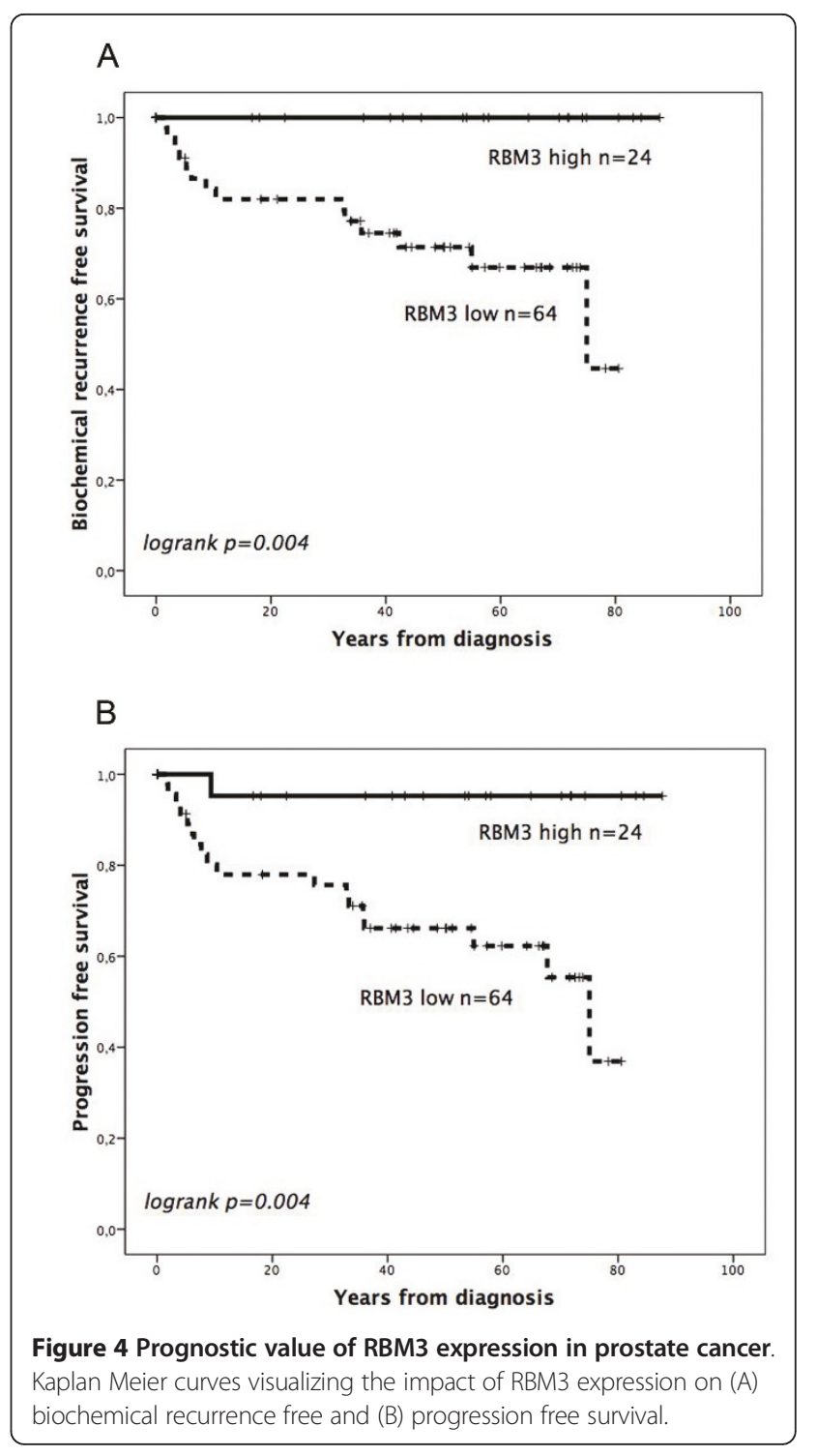

multiple cancer forms. The mechanisms behind these findings remain to be elucidated, an undertaking that might be somewhat challenging as current in vitro data point towards a proto-oncogenic role for RBM3 $[8,9,19]$ and, hence, do not readily seem to support the clinical situation. However, the common hypothesis that oncogene activation is associated with an aggressive tumour phenotype does not always hold true, as well exemplified by the association between microsatellite instability and good prognosis in colorectal cancer [20]. It is evident that RBM3, while sparsely expressed in normal tissue, is up-regulated in most cancer forms and their preinvasive stages in vivo. In light of the recently proposed association between RBM3 and DNA integrity in ovarian cancer [17], it could be speculated that RBM3 might play an important role in promoting early stages of tumourigenesis by interfering with the anti-cancer 
Table 1 Cox univariate and multivariate analysis of biochemical recurrence free survival and progression free survival according to RBM3 expression

\begin{tabular}{|c|c|c|c|}
\hline \multirow{2}{*}{\multicolumn{2}{|c|}{ RBM3 intensity*fraction dichotomized }} & \multicolumn{2}{|c|}{ Progression free survival } \\
\hline & & \multirow[t]{2}{*}{ HR $(95 \%$ Cl) } & \multirow[t]{2}{*}{$\mathrm{p}$-value } \\
\hline & $\mathrm{n}$ (events) & & \\
\hline \multicolumn{4}{|l|}{ Univariate } \\
\hline Low (0-2) & $64(18)$ & 1.00 & \\
\hline High (3-8) & $24(1)$ & $0.09(0.01-0.71)$ & 0.021 \\
\hline \multicolumn{4}{|l|}{ Multivariate } \\
\hline Low (0-2) & $64(16)$ & 1.00 & \\
\hline \multirow[t]{2}{*}{ High (3-8) } & $24(1)$ & $0.06(0.01-0.50)$ & 0.009 \\
\hline & & \multicolumn{2}{|c|}{$B C R$ free survival } \\
\hline \multicolumn{2}{|c|}{ RBM3 intensity*fraction continuous } & $\mathrm{HR}(95 \% \mathrm{Cl})$ & p-value \\
\hline & n (events) & & \\
\hline Univariate & $88(14)$ & $0.56(0.34-0.93)$ & 0.024 \\
\hline Multivariate & $88(12)$ & $0.41(0.19-0.89)$ & 0.024 \\
\hline
\end{tabular}

Multivariate analysis including adjustments for preoperative PSA level, Gleason score, presence or absence of extracapsular extension, seminal vesicle invasion and positive surgical margins.

barrier provided by various DNA damage checkpoint mechanisms [21-23]. On the other hand, once a tumour has been established, an attenuated capability of DNAdamage response caused by RBM3 over expression might hinder the pressure for selection of more malignant clones $[21,22]$. Further studies are warranted to explore these associations.

In this study, we used a well-validated monoclonal antibody for detection of RBM3 expression [15,16]. Immunohistochemistry has several advantages compared to other assays in that it can easily be incorporated into clinical protocols and allows for assessment of the subcellular localization of proteins, which might have important prognostic implications. Our results indicate that immunohistochemical assessment of RBM3 expression in formalinfixed paraffin embedded tumour samples could be a useful tool for prognostication and treatment stratification of prostate cancer patients. Since this was a small study these findings should be further validated in larger studies, preferably tumours from prospective, clinical trials.

\section{Additional material}

Additional file 1: Patient and tumour characteristics.

Additional file 2: Association between RBM3 and clinicopathological parameters.

\section{List of abbreviations}

BCR: biochemical recurrence; CRT: classification regression tree analysis; DNA: deoxyribonucleic acid; HR: hazard ratio; IHC: immunohistochemistry; NF: nuclear fraction; NI: nuclear intensity; NS: nuclear score; PIN: prostatic intraepithelial neoplasia; PSA: prostate specific antigen; RBM3: RNA-binding motif 3; RNA: ribonucleic acid; RRM: RNA recognition motif; TMA: tissue microarray.

\section{Acknowledgements}

This work was supported by grants from the Knut and Alice Wallenberg Foundation, the Swedish Cancer Society, Swedish Scientific Council, Gunnar Nilsson's Cancer Foundation, and the Research fund of Skåne University Hospital, Malmö.

We thank Elise Nilsson and Björn Nodin for excellent technical assistance.

\section{Author details}

'Department of Clinical Sciences, Pathology, Lund University, Skåne University Hospital, 22185 Lund, Sweden. 'Department of Clinical Sciences, Division of Urological Cancers, Lund University, Skåne University Hospital, 20502 Malmö, Sweden. ${ }^{3}$ Department of Proteomics, AlbaNova University Center, Royal Institute of Technology, 10691 Stockholm, Sweden. ${ }^{4}$ Science for Life Laboratory, Royal Institute of Technology, 10691 Stockholm, Sweden.

\section{Authors' contributions}

$L J$ carried out the immunohistochemical analysis, performed the statistical analysis, and drafted the manuscript. AG assisted with the statistical analysis and helped draft the manuscript. DU and $A B$ collected the clinical data. $A B$ also contributed with clinical input. MU participated in the conception and design of the study. KJ participated in the conception and design of the study, the immunohistochemical analysis and helped draft the manuscript. All authors read and approved the final manuscript.

\section{Competing interests}

The authors declare that they have no competing interests.

Received: 31 August 2011 Accepted: 28 September 2011

Published: 28 September 2011

\section{References}

1. Jemal A, Bray F, Center MM, Ferlay J, Ward E, Forman D: Global cancer statistics. CA Cancer J Clin 2011, 61(2):69-90

2. Ferlay J, Parkin DM, Steliarova-Foucher E: Estimates of cancer incidence and mortality in Europe in 2008. Eur J Cancer 2010, 46(4):765-781.

3. Bill-Axelson A, Holmberg L, Ruutu M, Garmo H, Stark JR, Busch C, Nordling S, Haggman M, Andersson SO, Bratell S, et al: Radical prostatectomy versus watchful waiting in early prostate cancer. N Engl J Med 2011, 364(18):1708-1717.

4. Pound CR, Partin AW, Eisenberger MA, Chan DW, Pearson JD, Walsh PC: Natural history of progression after PSA elevation following radical prostatectomy. JAMA 1999, 281(17):1591-1597.

5. Bjartell A, Montironi R, Berney DM, Egevad L: Tumour markers in prostate cancer II: diagnostic and prognostic cellular biomarkers. Acta Oncol 2011, 50(Suppl 1):76-84.

6. Shariat SF, Semjonow A, Lilja H, Savage C, Vickers AJ, Bjartell A: Tumor markers in prostate cancer I: blood-based markers. Acta Oncol 2011, 50(Suppl 1):61-75

7. Danno S, Nishiyama H, Higashitsuji H, Yokoi H, Xue JH, Itoh K, Matsuda T, Fujita J: Increased transcript level of RBM3, a member of the glycine-rich RNA-binding protein family, in human cells in response to cold stress. Biochem Biophys Res Commun 1997, 236(3):804-807.

8. Lleonart ME: A new generation of proto-oncogenes: cold-inducible RNA binding proteins. Biochim Biophys Acta 2010, 1805(1):43-52.

9. Sureban SM, Ramalingam S, Natarajan G, May R, Subramaniam D, Bishnupuri KS, Morrison AR, Dieckgraefe BK, Brackett DJ, Postier RG, et al: Translation regulatory factor $\mathrm{RBM} 3$ is a proto-oncogene that prevents mitotic catastrophe. Oncogene 2008, 27(33):4544-4556.

10. Wellmann S, Truss M, Bruder E, Tornillo L, Zelmer A, Seeger K, Buhrer C: The RNA-binding protein RBM3 is required for cell proliferation and protects against serum deprivation-induced cell death. Pediatr Res 2010, 67(1):35-41.

11. Jogi A, Brennan DJ, Ryden L, Magnusson K, Ferno M, Stal O, Borgquist S, Uhlen M, Landberg G, Pahlman S, et al: Nuclear expression of the RNAbinding protein RBM3 is associated with an improved clinical outcome in breast cancer. Mod Pathol 2009, 22(12):1564-1574. 
12. Uhlen $M$, Bjorling E, Agaton C, Szigyarto CA, Amini B, Andersen E, Andersson AC, Angelidou P, Asplund A, Asplund C, et al: A human protein atlas for normal and cancer tissues based on antibody proteomics. Mol Cell Proteomics 2005, 4(12):1920-1932.

13. Bjorling E, Lindskog C, Oksvold P, Linne J, Kampf C, Hober S, Uhlen M, Ponten F: A web-based tool for in silico biomarker discovery based on tissue-specific protein profiles in normal and cancer tissues. Mol Cell Proteomics 2008, 7(5):825-844.

14. Ponten F, Jirstrom K, Uhlen M: The Human Protein Atlas-a tool for pathology. J Pathol 2008, 216(4):387-393.

15. Ehlen A, Brennan DJ, Nodin B, O'Connor DP, Eberhard J, AlvaradoKristensson M, Jeffrey IB, Manjer J, Brandstedt J, Uhlen M, et al: Expression of the RNA-binding protein RBM3 is associated with a favourable prognosis and cisplatin sensitivity in epithelial ovarian cancer. J Trans/ Med 2010, 8:78.

16. Jonsson L, Bergman J, Nodin B, Manjer J, Ponten F, Uhlen M, Jirstrom K: Low RBM3 protein expression correlates with tumour progression and poor prognosis in malignant melanoma: An analysis of 215 cases from the Malmo Diet and Cancer Study. J Transl Med 2011, 9(1):114.

17. Ehlen O, Nodin B, Rexhepaj E, Brandstedt J, Uhlen M, AlvaradoKristensson M, Ponten F, Brennan DJ, Jirstrom K: RBM3-Regulated Genes Promote DNA Integrity and Affect Clinical Outcome in Epithelial Ovarian Cancer. Transl Oncol 2011, 4(4):212-221.

18. Cookson MS, Aus G, Burnett AL, Canby-Hagino ED, D'Amico AV, Dmochowski RR, Eton DT, Forman JD, Goldenberg SL, Hernandez J, et al: Variation in the definition of biochemical recurrence in patients treated for localized prostate cancer: the American Urological Association Prostate Guidelines for Localized Prostate Cancer Update Panel report and recommendations for a standard in the reporting of surgical outcomes. J Urol 2007, 177(2):540-545.

19. Zeng $Y$, Kulkarni P, Inoue T, Getzenberg RH: Down-regulating cold shock protein genes impairs cancer cell survival and enhances chemosensitivity. J Cell Biochem 2009, 107(1):179-188.

20. Popat S, Hubner R, Houlston RS: Systematic review of microsatellite instability and colorectal cancer prognosis. J Clin Oncol 2005, 23(3):609-618.

21. Bartkova J, Rajpert-De Meyts E, Skakkebaek NE, Lukas J, Bartek J: DNA damage response in human testes and testicular germ cell tumours: biology and implications for therapy. Int J Androl 2007, 30(4):282-291, discussion 291.

22. Bartkova J, Horejsi Z, Koed K, Kramer A, Tort F, Zieger K, Guldberg P, Sehested M, Nesland JM, Lukas C, et al: DNA damage response as a candidate anti-cancer barrier in early human tumorigenesis. Nature 2005, 434(7035):864-870.

23. Gorgoulis VG, Vassiliou LV, Karakaidos P, Zacharatos P, Kotsinas A, Liloglou T, Venere M, Ditullio RA Jr, Kastrinakis NG, Levy B, et al: Activation of the DNA damage checkpoint and genomic instability in human precancerous lesions. Nature 2005, 434(7035):907-913.

doi:10.1186/1746-1596-6-91

Cite this article as: Jonsson et al:: High RBM3 expression in prostate cancer independently predicts a reduced risk of biochemical recurrence and disease progression. Diagnostic Pathology 2011 6:91.

\section{Submit your next manuscript to BioMed Central and take full advantage of:}

- Convenient online submission

- Thorough peer review

- No space constraints or color figure charges

- Immediate publication on acceptance

- Inclusion in PubMed, CAS, Scopus and Google Scholar

- Research which is freely available for redistribution

Submit your manuscript at www.biomedcentral.com/submit 